\title{
Study of a Combination of Complex Function Learning with Mathematical Modeling
}

\author{
Pin-Chao MENG ${ }^{1, a}$, Zhi-Xia JIANG ${ }^{1, b, *}$, San-Zhi SHII, ${ }^{1, c}$, Shi-Ming LIANG ${ }^{1, d}$ \\ ${ }^{1}$ College of Science, Changchun University of Science and Technology, China \\ amengpc@cust.edu.cn, bzhixia_jiang@126.com, c'shisanzhi@sina.com \\ ${ }^{*}$ Corresponding author
}

Keywords: Education reform, Complex function teaching, Mathematical modeling, Innovation teaching.

\begin{abstract}
Complex function teaching essentially focuses on theoretical studying rather than practical application with respect to the properties of theoretical and abstract complex function course. This paper proposes a combination of mathematical modeling with complex function teaching, discusses a promising method of enhancing complex function teaching.
\end{abstract}

\section{Introduction}

"Complex function" is a basic specialty course in Electrical and Electronics majors. Plenty of science achievements are based on complex function[1,2,3]. Especially in recent decades, complex function methods have been expanded and improved over time with the rapid development of communication technology, which requires more talents to apply complex function theory and methods into engineering practice. Thus, a new challenge on complex function teaching has been issued.

Complex function curriculum involves Advanced Mathematics theories. This abstract and profound course requires solid foundation of mathematics and strong logical thinking, making it tougher to understand $[4,5,6]$. Students basically memorize certain formulas and theorems, which barely sharpen their skills to figure out the practical problems. Therefore, both the theories and practice, including the utilize of multimedia teaching, traditional teaching and Matlab supplementary means, are required to be emphasized to improve the quality of teaching by research of teaching reforms. The idea of integrating mathematical modeling into teaching may brighten the class as well as enhance the teaching effect. This article discusses the necessity of the combination of complex function teaching and mathematical modeling with respect to current issues, and comes up with an explicit way of mathematical modeling teaching and corresponding problems.

\section{Necessity of mathematical modeling ideas in Complex function teaching}

Complex function is an applied based course. However, the teaching content is somewhat disconnected with its strong applicability in current teaching. Class lectures merely focus on the basic idea, methodology and theories, while ignoring the relevant theory application background, the students hence cannot solve the practical problem seven they are equipped with theories. For instance, analytic functions and harmonic functions are fundamental knowledge in complex functions, but students barely know their real senses and uses in two-dimensional electrostatic fields. Furthermore, assuming that teachers make up a two-dimensional practical problem; does a student manage to employ a two-dimensional Laplace equation to illustrate it? Thus, students application skills can hardly get improved and strengthened, and they may also lose interest in this type of teaching mode.

Lectures deviating practices are inappropriate to cultivate students' application ability, then how to effectively deal with the problems of complex function and integral transform teaching? Introducing mathematical modeling idea is clearly effective. When talking about the theoretical 
stuff, instructors may make use of mathematical methods, combing with some application backgrounds and prospects, selecting some appropriate examples. Thus, the students may have a general idea about the combination of theories and realistic application; correspondingly, they are capable to transfer the practical problems into mathematical theories. This teaching mode could meet the requirements of the times, and cultivate the talents with solid theoretical basis as well as applications and innovative abilities.

The nature of mathematical modeling is to apply mathematical theories to describe the objective rules in practical problems, thereby providing a scientific direction to social production and life. The idea of integrating mathematical modeling into complex function and integral transform teaching, can not only arouse students' enthusiasm adequately, but also help them to understand some possible applications and senses of the subject, sharpening their modeling skills. Therefore, integrating mathematical modeling to "Complex Function" courses does make perfect sense.

\section{Combine ideas of mathematical modeling into Complex Function course}

In lectures, how to select the right access points to convey the idea of mathematical modeling so that the integrity of knowledge is ensured, and students' modeling thinking is also developed? In the actual teaching process, we made some innovation teaching contents, methods, and exams. We achieved great teaching results.

\section{Update teaching contents with mathematical modeling examples}

Complex Variables and integral transformation have a wide range of applications in many fields. Therefore, they can be presented in the class with more mature, more cutting-edge application results or specific examples. Given the level of awareness of students' mental content and curriculum, thoughts, and methods, selection of examples should be simple ones instead of complicated ones. Examples of complex functions should not only reflect the core knowledge of integral transformation, but also they should be easy to understand and stimulate students' interest in learning. Students could clearly feel that these applications are all around us in our daily lives. This requires teachers to select the appropriate model instance and teaching contents, with applicability and practicality highlighted, so that we can achieve the purpose of training talents for real-world applications.

In lectures of concept of complex, we introduced camera as an example. A camera is typically divided into two types, optical camera and digital camera. Digital cameras are generally installed by adding Bayer filter on Charge-coupled Device (CCD). CCD is an integrated circuit with a number of rows of the capacitors sensing light. These capacitors can convert the image into a digital signal. The technology of direct processing CCD camera image has greatly advanced optical holographic technology. Light waves that were normally only obtained by phase-shift now can be extracted through digital reconstruction phase. The reconstructed light wave is a complex signal, through which we can retrieve each point's phase and light intensity. We can then use Fourier transform of complex signal to process digital holographic interferometry methods. Such examples can not only embrace real-life experience of students into learning, broadening their views, but also pave a way for students for subsequent sections.

After the lecture of integral of complex functions, we provide a model example for students to discuss: how to measure the temperature of the inner earth? We inspired and guided students that they can measure the temperature of some surface points of the Earth, combined with Cauchy integral formula, to calculate temperature of geocentric Earth center. Using this modeling example, we could help students to get rid of the style of mechanical calculation only using the formula. Students were able to realize that basic theories and formulas in solving real-life problems are also very practical.

Of course, the closer the problems are to real world, the more interesting they are for students. For instance, when receiving a text message, every one wishes to get the message with complete information intact. But sometimes a text message may contain some garbled or missing characters. 
If we simply treat edited characters as an area and the transmission of the message as the transmission of the area, signal transmission can be considered as mapping in math. This kind of mapping is conformal mapping in complex functions. It is a mapping that maps an area to another area, keeping some of their properties unchanged. This model example can be integrated into lecture and the original boring classes can be vivid now. And students' interests and initiative is fully mobilized by the example.

\section{Reforming teaching method to combine mathematical modeling ideas}

Combining mathematical modeling examples in lectures of complex functions and integral transform can not only stimulate students' enthusiasm of active learning, but also guide them to integrate theory with practice by mastering modeling ideas. It can prompt students to develop mathematical modeling thoughts to solve practical problems, so as to improve modeling capabilities and enhance training for application-oriented talents.

In lectures of examples of complex functions and integral transform models, we can use improved teaching methods to let students be masters of the class. We can use model group discussion to inspire and help students to analyze and solve problems. To better teach mathematical modeling ideas, first we need to emphasize on how to describe real-world problems with mathematical languages. Second, we would emphasize on which theories students need to construct complex function models. Third, we elaborate on which methods or solutions in complex functions can solve the problem. In the whole process, we focused on "problem background - model construction - result analysis - result application" to guide students to learn theories of complex functions and solve or explain practical problems with these theories on purpose.

\section{Conclusion}

Teaching is an organic dialectical unity process that involves both instructions of teachers and learning of students. "Teaching a man how to fish is better than giving the man a fish." To train highly qualified professionals, teachers should not only focus on "teaching", but also should guide students to learn. Developing and mastering the idea of mathematical modeling is as important as learning theories of complex functions. It is a long, gradual process to seamlessly convey the ideas of mathematical modeling in teaching. In future lectures, teachers need to continue to explore more and better ways to integrate the ideas of mathematical modeling and constantly improve the teaching methods of complex functions and integral transform curriculum.

\section{References}

[1] WANG Zhiliang, SHEN Lin-fang, YAO Ji, GAO Chengjie, Shallow buried tunnel surrounding rock stress field calculation of complex function method, Rock and Soil Mechanics, 31(s1), 86-90, 2010.

[2] WANG Xiaofang, YAN Guangrong, LEI Yi, Complex variable function model and edge detection of color images, Opto-Electronic Engineering,35(2), 90-96, 2008.

[3] LIU Yunfang, LIU Yuankun, XU Jing, Non circular cavity stress monitoring feedback analysis method of complex function, Chinese Journal of Geotechnical Engineering, 34(10), 1925-1930, 2012.

[4] DENG Yingdong , Lu Yanming, The teaching reform for the complex variable function and integral transform, Journal of Nantong Institute of Technology, 21(4), 2004.

[5] Cao Lixia, Tian Shujie, Zhao Zhongkui, Discussion of University Mathematics Education, Education Teaching Forum, 4(22), 51-53, 2012.

[6] Wang Shuyan, Reflections on teaching reform of "the Function of Complex Variable and 
Integral Transform", Journal of Northeast Agricultural University (Social Science Edition), 9(3),2011. 